\title{
Catalytic Growth and Properties of Carbon Nanotubes from Fe-Mo/MgO by Chemical Vapor Deposition
}

\author{
Jong-Chang Woo, Kyoung-Tae Kim, Gwan-Ha Kim, Jong-Sik Kim, \\ Jong-Gyu Kim, and Chang-Il Kim ${ }^{\mathrm{a}}$ \\ School of Electrical and Electronic Engineering, Chung-Ang University, \\ 221 Heukseok-dong, Dongjak-gu, Seoul 156-756, Korea \\ ${ }^{a}$ E-mail : cikim@cau.ac.kr
}

(Received July 27 2007, Accepted September 10 2007)

\begin{abstract}
Carbon nanotubes (CNTs) are largely synthesized on Fe catalysts by catalytic chemical vapor deposition. The various shapes and compositions of these nanostructure CNTs were obtained by controlled parameters such as the reaction temperature, gas-mixing ratio. The influence of these parameters is investigated, together with observations of the produced materials after the purification processes. A diameter of CNTs, range from 2 to $10 \mathrm{~nm}$, closely correlated with the size of the catalyst particle found attached to the tube end. The yield of CNTs was estimated to be $88.5 \%$ and the purities of CNTs thus obtained were more than $80 \%$. The experimental results were documented with field emission scanning electron microscopy and raman spectroscopy and transmission electron microscopy, both before and after the purification.
\end{abstract}

Keywords : Carbon nanotubes, Growth, High density, Catalytic CVD

\section{INTRODUCTION}

The remarkable physical, chemical and electrical properties of CNTs make them attractive possibilities for many microelectronic application areas and nanoscale devices. Since the CNTs were firstly discovered in 1991, extensive interests were focused on the fabrication of CNTs. In recent several years, due to its low cost and large-scale production capacity, the catalytic decomposition of hydrocarbon has become one of the most important synthesis methods than arc discharge, laser abalation and chemical vapor deposition (CVD) for carbon micro- and nano-structures. Dai[1] first synthesized CNTs by CVD on a $\mathrm{Mo} / \mathrm{Al}_{2} \mathrm{O}_{3}$ catalyst with $\mathrm{CO}$ as the carbon feedstock, but the yield was very low. Recently, several studies [2-6] have demonstrated that the catalytic method could be a possibility for the selective and controlled grown CNTs on various catalysts and support materials and high-quality of CNTs at relatively low cost. All of those literatures indicated that the yield and quality of CNTs are very sensitive to catalyst preparation.

In general, silicon oxide, magnesia and alumina have all been employed as supporting materials. These materials were easily aggregated and the effective catalyst area was decreased, leading to a decrease in the yield. We also employed PEG 200 among the organic additives such as citric acid and poly-(ethylene glycol) for solving the upper problems. In fact, it is the most effective as a dispersant at improving the yield of CNTs due to the effect of additives on the specific surface area of the catalyst[7]. The CNTs were extracted by dissolution of $\mathrm{MgO}$ and part of the $\mathrm{Fe}$ catalyst in the PEG 200 aqueous solution. In addition, MgO presents the advantage over the supports such as alumina, spinel, and silica that it can be readily dissolved in acids. Therefore, the produced CNTs can be removed easily from support by a simple acidic treatment to obtain a sample containing high yields of CNTs (70-80\%).

In this work, we reported an improved CVD method for preparation of CNTs with high productivity using a novel aerogel supported $\mathrm{Fe}-\mathrm{Mo} / \mathrm{MgO}$ catalyst. High qualities of CNTs can be synthesized by catalytic decomposition of methane and acetylene over welldispersed metal particles supported on $\mathrm{MgO}$. In order to achieve the highest yield and quality and the highest purity of CNTs, we investigated in detail the effect on the growth temperature and reaction gas among the many growth parameters. Moreover, we adopt a purification process to raw CNTs synthesized by CVD. The yield, the quality and the quantity of CNTs have been investigated by Raman spectroscopy, fieldemission scanning electron microscopy and TEM, both before and after the purification. 


\section{EXPERIMENTAL}

\subsection{Catalyst preparation}

The $\mathrm{Fe} / \mathrm{Mo} / \mathrm{MgO}$ catalysts were prepared by a wet mechanical mixing and combustion method[8]. The weighted amounts of compounds $\left(\mathrm{NH}_{4}\right)_{6} \mathrm{Mo}_{7} \mathrm{O}_{24} \cdot 4 \mathrm{H}_{2} \mathrm{O}$ (99.0\%, Samchun), $\mathrm{Mg}\left(\mathrm{NO}_{3}\right)_{2} \cdot 6 \mathrm{H}_{2} \mathrm{O}$ (98.0\%, Samchun), and $\mathrm{Fe}\left(\mathrm{NO}_{3}\right)_{3} \cdot 9 \mathrm{H}_{2} \mathrm{O}(99.99 \%$, Aldrich) were dissolved in the minimum amount polyethylene glycol 200 (PEG 200) to yield the molar ratios as in $\mathrm{Fe}$ : Mo: $\mathrm{MgO}=1: 0.05: 13$ were mixed together. Then the above solution were transferred to a quartz boat, and directly brought into a furnace, heated to $550{ }^{\circ} \mathrm{C}$, where it ignited and stayed on furnace for about $15 \mathrm{~min}$. The boat was taken out and cooled to room temperature. The catalyst prepared this way is in the form of very fine powder with surface areas $\sim 600 \mathrm{~m}^{2} / \mathrm{g}$. The powder was calcined at $500{ }^{\circ} \mathrm{C}$ in air for $30 \mathrm{~min}$ before used for carbon nanotubes growth.

\subsection{Carbon nanotube growth}

The synthesis of CNTs was carried out in a simple CVD setup made of a horizontal quartz tube furnace and gas flow control system. In a typical growth experiment is as follow: $200 \mathrm{mg}$ catalyst power was uniformly put into an alumina boat inside a quartz tube, and when the furnace reached the reaction temperature, $800^{\circ} \mathrm{C}$ to 1000 ${ }^{\circ} \mathrm{C}$, the quartz boat was inserted into the center of reactor while the nitrogen as the protective gas flow was maintained for $5 \mathrm{~min}$. Subsequently, a mixture of $\mathrm{CH}_{4}: \mathrm{H}_{2}=75 / 300$ or $\mathrm{C}_{2} \mathrm{H}_{2} / \mathrm{N}_{2} / \mathrm{H}_{2}=50 / 1000 / 25$ or $\mathrm{C}_{2} \mathrm{H}_{2} / \mathrm{N}_{2}=$ $50 / 1000$ was introduced. The growth reaction was maintained for $30 \mathrm{~min}$. After methane and hydrogen flow was turn off and nitrogen flow turn on and temperature reduced to room temperature. The black materials were then weighted and characterized.

\subsection{Carbon nanotube purification}

In order to eliminate the metallic catalysts and the amorphous carbon, we adopt the following purification procedures to as-grown products synthesized by above catalytic CVD. The purification procedure of as-grown products is as follows; the sample was refluxed in $30 \%$ nitric acid at $100{ }^{\circ} \mathrm{C}$ for $12 \mathrm{~h}$. The sample was washed thoroughly with deionized water, the as-grown products were immersed in $6 \mathrm{M}$ hydrochloric acid for $24 \mathrm{~h}$. These two processes were repeated several times until the color of acid was unchanged, respectively. The sample was washed thoroughly with deionized water. After rinsing and drying, the black sediment was finally obtained.

\subsection{Characterization}

As-prepared samples were characterized by field emission scanning electron microscope (FESEM) [JEOL, JSM-6700F]. Raman spectroscopy [Burker, RFS 100/S] which was equipped with an $\mathrm{Nd}$ :YAG laser with an excitation wavelength of $1064 \mathrm{~nm}$ was used to demonstrate

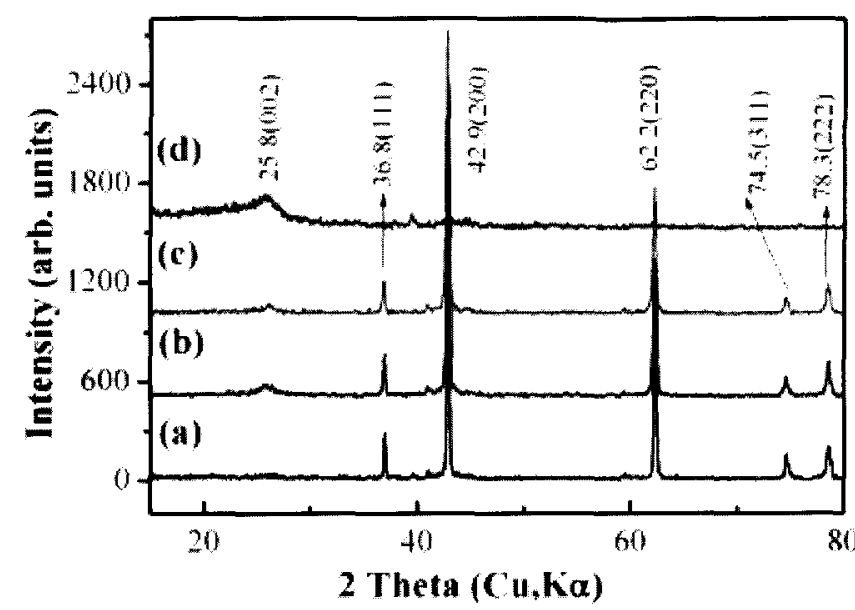

Fig. 1. XRD pattern of as-synthesized carbon nanotubes (a) at $1000^{\circ} \mathrm{C}$, (b) at $900{ }^{\circ} \mathrm{C}$, (c) at $800^{\circ} \mathrm{C}$, and (d) the purified carbon nanotubes.

the diameter distribution of CNTs. This material was sonicated with methanol, and a drop place on a holey carbon grid for observation in the transmission electron microscope (TEM). Themogravimetric analysis (TGA) [TA Instrument, TGA2050] was used to investigate weight change and the content of carbon in the sample.

\section{RESULTS AND DISCUSSION}

Figure 1 is shown the XRD pattern for as-synthesized and purified samples. The difference between the assynthesized and the purified CNTs has been investigated. As shown in Fig. 1(a)-(c), it was shown the diffraction peak of CNTs, graphite, and metal particles. A sharp diffraction peak at $2 \theta=25.8^{\circ}$ with a d-value of 0.345 $\mathrm{nm}$ is from graphite. The peak at $2 \theta=36.8,42.9,62.2$, 74.5 and 78.3 corresponds to $\mathrm{MgO}$. Unfortunately, unwanted impurities may be included in the CNTs during their synthesis. These impurities include amorphous carbon, fullerenes, and the remains of primary materials. The XRD profile is shown Fig. 1(d) corresponds to the sample of CNTs after purification process. It presents some change, the catalyst peaks nearly disappeared after acid treatment but a small quantity of peaks still remained. It was considered due to graphite layers which have resistance to the $\mathrm{HNO}_{3}$ acid; therefore, a small amount of encapsulated carbon coexists in the samples.

Figure 2(a)-(c) is shown the typical FESEM images of the as-synthesized carbon materials produced by catalytic reaction of $\mathrm{CH}_{4}$ over $\mathrm{Fe}-\mathrm{Mo} / \mathrm{MgO}$ catalyst at a growth temperature range, from $800{ }^{\circ} \mathrm{C}$ to $1000{ }^{\circ} \mathrm{C}$. Synthesized carbon filaments are mostly intricate and 

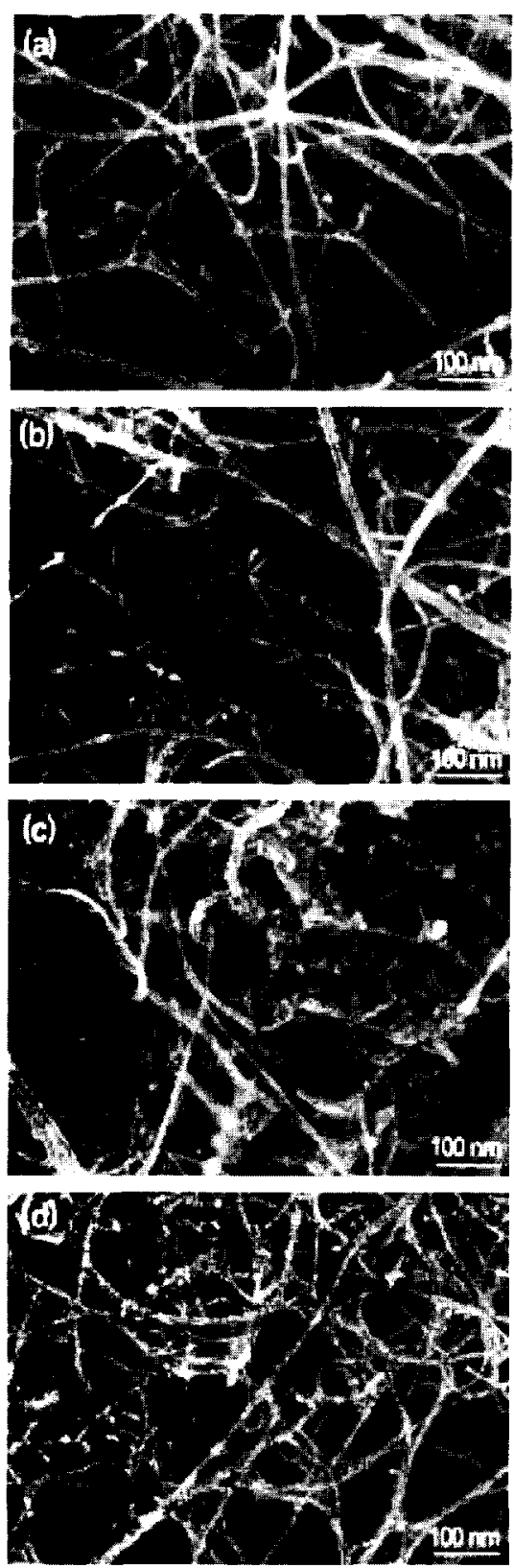

Fig. 2. FESEM images of grown CNTs (a) at $1000{ }^{\circ} \mathrm{C}$, (b) at $900{ }^{\circ} \mathrm{C}$, (c) at $800^{\circ} \mathrm{C}$, and (d) the purified carbon nanotubes.

bundle up together. As shown in the SEM images, we could find the effect of the growth temperature on the structure of CNTs. In a reaction temperature range of 900-1000 ${ }^{\circ} \mathrm{C}$, the morphology of the as-synthesized CNTs was almost similar, while the yield of CNTs was slightly increased with increasing reaction temperature. However, the yield of CNTs rapidly decreases and amorphous carbon materials were appeared in the carbon product when the reaction temperature was $800^{\circ} \mathrm{C}$. To quantify the yield, we conducted the weight gain measurement. For a $30 \mathrm{~min}$ growth experiment, the yield of CNTs defined as the weight gain ratio of the produced
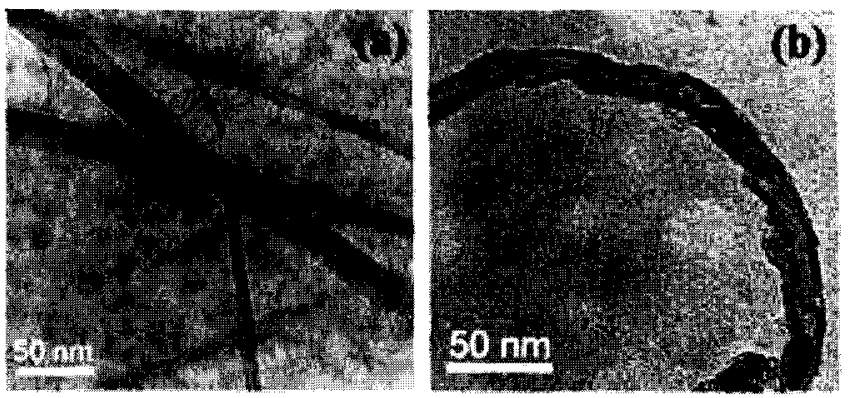

Fig. 3. TEM images of carbon nanotubes grown at 1000 ${ }^{\circ} \mathrm{C}$ for 30 min under a flow of $\mathrm{CH}_{4} / \mathrm{H}_{2} / \mathrm{N}_{2}$ (a) body, and (b) tip, respectively.

carbon bundles to the starting catalyst materials including support materials was about $108.28 \%, 96.9 \%$, and $88.5 \%$ in a reaction temperature at $1000{ }^{\circ} \mathrm{C}, 900{ }^{\circ} \mathrm{C}$, and $800{ }^{\circ} \mathrm{C}$, indicating a high yield of carbon filament, respectively. FESEM images of purified CNTs (see in Fig. 1(d)) indicated that most of amorphous carbon was dramatically decreased during purification process and the yield of CNTs was increased significantly. As a result of purification process, the weight of the soot sample was reduced to about $10 \mathrm{wt} . \%$ of the original sample and the yield of $96.6 \mathrm{wt} . \%$ of as-grown samples.

TEM was employed to further characterize the tubular structure of the nanotubes grown using catalytic CVD. Figure 3 shows cross-sectional TEM (200 kV, CM-20) images of carbon nanotubes grown at $1000{ }^{\circ} \mathrm{C}$ for 30 min under a flow of $\mathrm{CH}_{4} / \mathrm{H}_{2} / \mathrm{N}_{2}$. The nanotubes (Fig. 3(a)) exhibit exclusively a multi-walled bamboo-like structure with a central hollow region. The closed tips (Fig. 3(b)) are encapsulated catalytic particles and the curvature of compartment layer is oriented toward the closed tip. The tip growth model is adequate to describe these results. The catalytic particles are attached to the tips of nanotubes.

Figure 4 is the high-resolution TEM (HRTEM, $300 \mathrm{kV}$, JEM-3000F) result showing the interior, wall structures (Fig. 4(a)) and tip (Fig. 4(b)) of nanotubes grown at 1000 ${ }^{\circ} \mathrm{C}$ for 30 min under a flow of $\mathrm{CH}_{4} / \mathrm{H}_{2} / \mathrm{N}_{2}$. The fringes in a multi-wall are individual cylindrical graphitic layers. The lined part is nearly $3.06 \mathrm{~nm}$ and this has 9 graphitic walls. As can be seen in Fig. 4(a), the nanotubes have outer diameters of about $10 \mathrm{~nm}$ and inner diameters of $2 \sim 3 \mathrm{~nm}$. This is clearly consistent with the fact that the graphite spacing is $\mathrm{c} / 2=0.34 \mathrm{~nm}$. High resolution TEM of purified CNTs indicated that even after $\mathrm{HNO}_{3}$ and $\mathrm{HCl}$ treatments the tubes existed in the form of bundles. General quality of the tubes was found to be good, however, with a very few defects. The tube surfaces were not found to be associated with any amorphous carbon. In general, the number of walls of the tubes was 2-25 with an average outer and inner diameter of 4.8$10.1 \mathrm{~nm}$, respectively. 


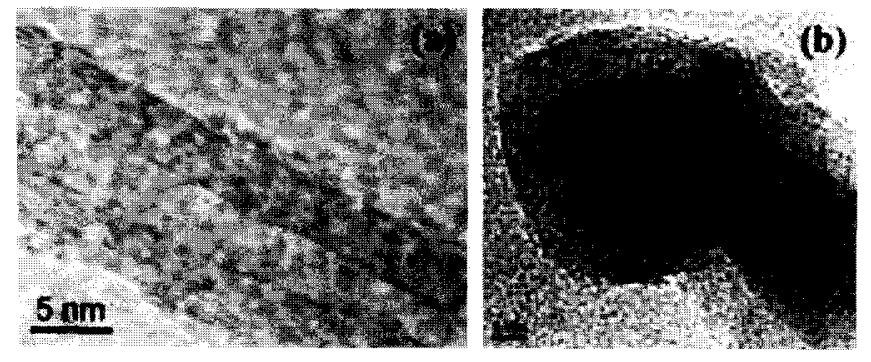

Fig. 4. HRTEM images of carbon nanotubes grown at $1000{ }^{\circ} \mathrm{C}$ for 30 min under a flow of $\mathrm{CH}_{4} / \mathrm{H}_{2} / \mathrm{N}_{2}$ (a) body, and (b) tip, respectively.

The Raman scattering spectra of the synthesized and the purified CNTs are shown in Fig. 5. The difference in microstructure between the as-synthesized CNTs and the purified CNTs has been further investigated by raman spectroscopy. The spectrum corresponds to a typical raman spectrum of the as-grown materials produced at three different growth temperatures and the purified CNTS, respectively. The G-band (observed at $1590 \mathrm{~cm}^{-1}$ ) is the strongest peak. This very narrow and strong peak associated with the G-band indicating that structural defects along the tube wall are low. The peak associated with the D-band $\left(1276 \mathrm{~cm}^{-1}\right)$ is related to the level of disorder carbon. Its strength can be either identified with wall disorder or with the presence of carbon coating the outside of tubular bundles. The low $I_{D} / I_{G}$ values indicate that the CNTs have a perfect graphite structure. The relatively weak D-band compare to G-band demonstrates that the as-synthesized samples contain small amount of amorphous carbon materials, revealing that high-purity CNTs are synthesized in our method. As shown in Fig. 5(a), several peaks are observed in the spectrum: 104$264 \mathrm{~cm}^{-1}$. These peaks are presented the radial breathing mode (RBM) in the $50-300 \mathrm{~cm}^{-1}$ range, which is well known to be strongly dependent on the diameters of CNTs. We used the equation as follows: $\omega\left(\mathrm{cm}^{-1}\right)$ $=12.5+223.5 / \mathrm{d}(\mathrm{nm})$ can be used to calculate the CNT diameters because the as-synthesized CNTs are packed into a bundle due to van der Waals interaction. According to this formula, the RBM peaks corresponding to the CNTs with inner diameters of from 0.41 to $1.13 \mathrm{~nm}$ were calculated. Fig. 5(d) is shown the Raman spectra of purified CNTs. The intensities of the disorder line (so called D-band) were relatively decreased compare to the as-grown CNTs (as shown Fig. 5(a)), while it appears that the RBM mode was changed. Through the reference[9] it is possible to assign an individual $\mathrm{RBM}$ mode in the Raman spectra to a particular $E_{11}$ band gap energy.

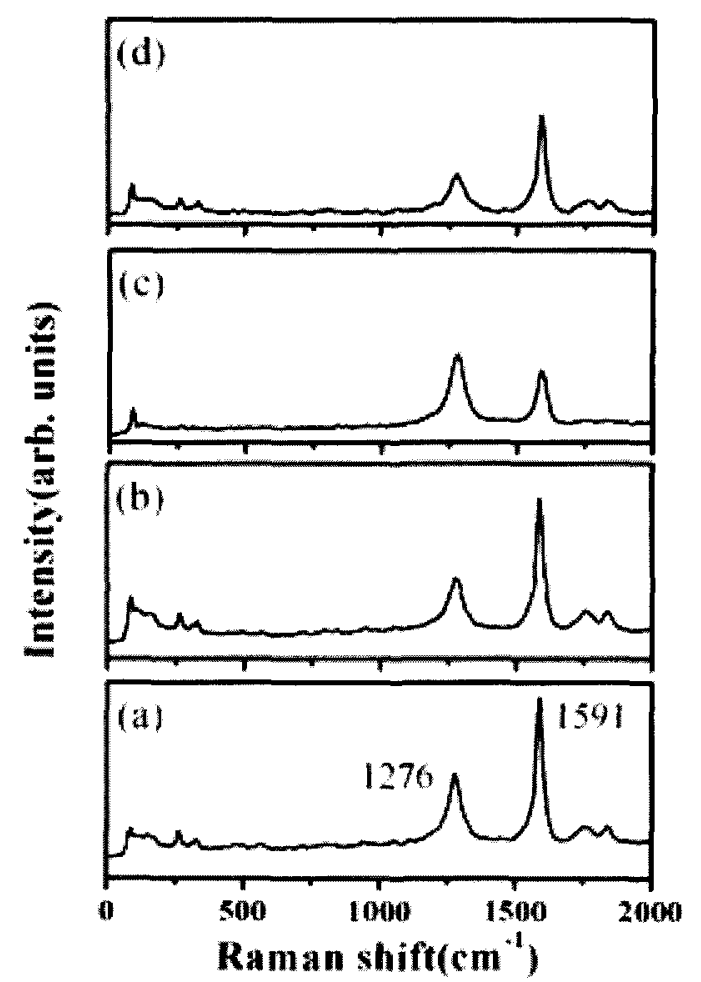

Fig. 5. Raman spectrum of as-synthesized carbon nanotubes (a) at $1000{ }^{\circ} \mathrm{C},\left(\right.$ b) at $900{ }^{\circ} \mathrm{C},(\mathrm{c})$ at $800^{\circ} \mathrm{C}$, and (d) the purified carbon nanotubes.

Figure 6 is shown the TGA graphs of the as-prepared products and the purified samples. Thermogravimetric analysis was performed in an oxidation environment to evaluate the resistance of different carbon materials toward oxidization. The sample was oxidized at temperature of $450-600{ }^{\circ} \mathrm{C}$. The maximum oxidation rate of the as-synthesized sample is found at around $590{ }^{\circ} \mathrm{C}$, while for the purified sample the maximum rates appear almost identically at $530{ }^{\circ} \mathrm{C}$. When the oxidation is carried out up to $1000^{\circ} \mathrm{C}$, an interesting phenomenon is noticeable for carbon materials. According to the literature[10], around $330{ }^{\circ} \mathrm{C}$ is a temperature to burn out amorphous carbon, $500-600{ }^{\circ} \mathrm{C}$ is for SWNTs, and 700 ${ }^{\circ} \mathrm{C}$ is for MWNTs. Therefore, our results can be a promising way to large-scale the synthesis of carbon nanotubes. Moreover, it is well known that DTG (Derivative TG) is a common method for quantitative analysis, the content of carbon materials corresponding to its peak area in DTG. Thus, it can be calculated that the yield of nanotubes is $85 \%$ in the raw produced with $\mathrm{Fe}-\mathrm{Mo}$ as catalyst. From above these results, our results show a promising technique for large-scale synthesis of carbon nanotubes which is relatively simple and effective. 


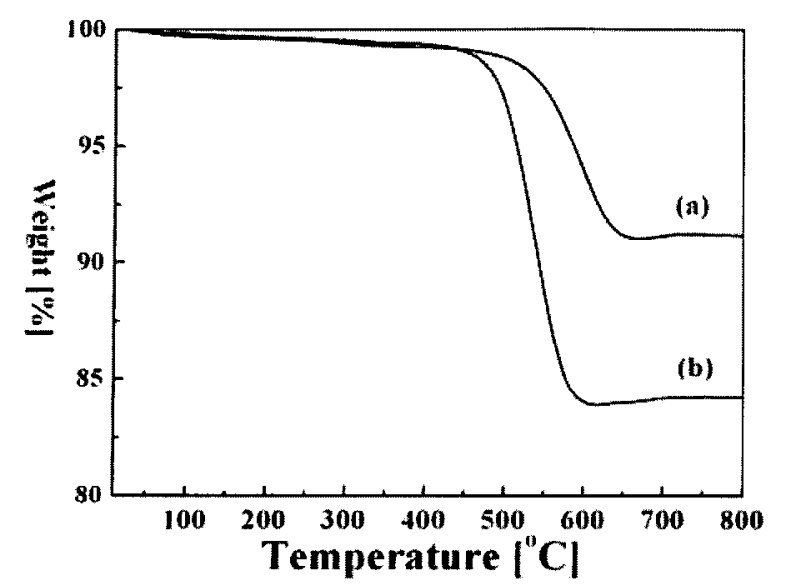

Fig. 6. Typical TGA curves for (a) the synthesized carbon nanotubes at $1000{ }^{\circ} \mathrm{C}$ and (b) the purified carbon nanotubes.

\section{CONCLUSION}

In this paper, we have shown that the large-scale production of CNTs is possible by catalytic chemical vapor deposition method similar to the two techniques, arc discharge and laser evaporation. Indeed, the method using catalytic decomposition of methane over welldispersed metal particles supported on $\mathrm{MgO}$ at $1000{ }^{\circ} \mathrm{C}$ has been adapted to produce large quantities of CNTs. we have successfully produced CNTs by catalytic decomposition of $\mathrm{CH}_{4}$ over $\mathrm{Fe}$ particles dispersed on porous $\mathrm{MgO}$ nanoparticles in high yield of above $88.5 \%$. The inner diameters of CNTs are in the range of $2-3 \mathrm{~nm}$ and outer diameters of about $10 \mathrm{~nm}$. The yield of CNTs was slightly increased with increasing reaction temperature, but the yield of CNTs rapidly decreases and amorphous carbon materials were appeared in the carbon product when the reaction temperature was $800^{\circ} \mathrm{C}$. The synthesis yield was estimated for removal of support by a simple purification process. The purities of the CNTs produced were estimated approximately to be of the order of $83 \%$. In the near future, these needs to be investigated and the purification of CNTs produced will be optimized to obtain pure CNTs in large quantities. And other reaction conditions of CNTs production, i.e., increasing (until $1200{ }^{\circ} \mathrm{C}$ ) and decreasing the reaction temperature, under $\mathrm{CH}_{4} / \mathrm{H}_{2}$, will be further investigated to improve the quantity and enhance the yield of CNTs.

\section{ACKNOWLEDGMENTS}

This Research was supported by the Chung-Ang University Research Grants in 2006.

\section{REFERENCES}

[1] H. Dai, A. G. Rinzler, P. Nikolaev, A. Thess, D. T. Colbert, and R. E. Smalley, "Single-wall nanotubes produced by metal-catalyzed disproportionation of carbon monoxide", Chem. Phys. Lett., Vol. 260, p. 471, 1996.

[2] Y. Yonglai, X. Hengyong, and L. Wenzhao, "Morphology observation of carbon deposition by $\mathrm{CH}_{4}$ decomposition over Ni-based catalysts", Nanotechnology, Vol. 16, p. 129, 2005.

[3] H. Kathyayini, I. Willems, A. Fonseca, J. B. Nagy, and N. Nagaraju, "Catalytic materials based on aluminium hydroxide, for the large scale production of bundles of multi-walled (MWNT) carbon nanotubes", Catal. Comm., Vol. 7, p. 140, 2006.

[4] B. C. Liu, S. C. Lyu, S. I. Jung, H. K. Kang, C. -W. Yang, J. W. Park, C. Y. Park, and C. J. Lee. "Single-walled carbon nanotubes produced by catalytic chemical vapor deposition of acetylene over Fe-Mo/MgO catalyst", Chem. Phys. Lett., Vol. 383, p. 104, 2004.

[5] E. Flahaut, Ch. Laurent, and A. Peigney, "Catalytic CVD synthesis of double and triple-walled carbon nanotubes by the control of the catalyst preparation", Carbon., Vol. 43, p. 375, 2005.

[6] M. Yamada, M. Kawana, and M. Miyake, "Synthesis and diameter control of multi-walled carbon nanotubes over gold nanoparticle catalysts", Appl. Cata. A; General., Vol. 302, p. 201, 2006.

[7] L. Shen, X. Zhang, Y. Li, X. Yang, J. Luo, and G. $\mathrm{Xu}$, "Effect of organic additives in catalyst preparation on the growth of single-wall carbon nanotubes prepared by catalyst-assisted chemical vapour deposition", Nanotechnology, Vol. 15, p. 337, 2004.

[8] K. C. Patil, "Advanced ceramics: combustion synthesis and properties Bull", Mater. Sci., Vol. 16, p. 533, 1993.

[9] S. M. Bachilo, M. S. Strano, C. Kittrell, R. H. Hauge, R. E. Smalley, and R. B. Weisman, "Structureassigned optical spectra of single-walled carbon nanotubes", Science, Vol. 298, p. 2361, 2002.

[10] A. G. Rinzler, J. Liu, and H. Dai, "Large-scale purification of single-wall carbon nanotubes: process, product, and characterization", Appl. Phys. A., Vol. 67, p. 29, 1998. 